\title{
ESTUDO DA EMISSÃO POR FLUORESCÊNCIA DE ULTRAVIOLETA NA QUANTIFICAÇÃO DE UM INIBIDOR DE CORROSÃO DO TIPO SAL DE AMÔNIO QUATERNÁRIO EM ÁGUA
}

\author{
Herllaine de Almeida Rangel* \\ Instituto Estadual do Ambiente, Secretaria Estadual do Ambiente, Governo do Estado do Rio de Janeiro, Av. Salvador Allende, \\ 5500, 22780-160 Rio de Janeiro - RJ, Brasil \\ Fábio Merçon \\ Instituto de Química, Universidade do Estado do Rio de Janeiro, Rua São Francisco Xavier, 524, Pavilhão Haroldo Lisboa da \\ Cunha, 20550-013 Rio de Janeiro - RJ, Brasil
}

Recebido em 22/2/11; aceito em 16/2/12; publicado na web em 30/4/12

\begin{abstract}
STUDY OF ULTRAVIOLET FLUORESCENCE EMISSION IN THE QUANTIFICATION OF CORROSION INHIBITOR OF THE QUATERNARY AMMONIUM SALT TYPE IN WATER. Quaternary ammonium salts are the corrosion inhibitors most frequently used by the oil industry. In this study, the ultraviolet fluorescence technique was evaluated for the analysis of a quaternary ammonium salt in water as a corrosion inhibitor. The comparison with standard salt showed that an alkyl aryl quaternary ammonium salt is the main fluorophore, with emission maxima at 306 and $593 \mathrm{~nm}$. The best instrumental parameters were: width of excitation and emission slits of 10 and $15 \mathrm{~nm}$, respectively, and scan rate of $10 \mathrm{~nm} \mathrm{~min}^{-1}$. The presence of aromatic compounds and biocides affects the analysis of corrosion inhibitors.
\end{abstract}

Keywords: corrosion inhibitor; quaternary ammonium salts; fluorescence.

\section{INTRODUÇÃO}

A retirada do petróleo do interior das rochas e o seu posterior processamento primário corresponde ao que se chama de produção dentro da indústria do petróleo. Nesta etapa, são utilizados produtos químicos para atender a demanda dos métodos cada vez mais sofisticados de produção e para solução de problemas operacionais, como a formação de espuma, emulsões, depósitos diversos e corrosão. A consequência deste fato é a geração de uma corrente aquosa, denominada água produzida, que consiste em uma mistura complexa. Para manter as condições de pressão na rocha-reservatório, normalmente é injetada água nas camadas inferiores do reservatório, o que contribui significativamente no volume total de água produzida. A composição da água produzida varia em função da vida do campo, cujo volume tende a crescer à medida que o campo envelhece. No Mar do Norte, por exemplo, a quantidade de água produzida com óleo é normalmente baixa (menos de $1 \%$ por volume) no início da produção, mas aumenta gradualmente, atingindo valores superiores a $80 \%$. $^{1-4}$

Dentre os produtos químicos de elevada importância utilizados durante a produção estão os inibidores de corrosão, que são aplicados desde o poço até os sistemas de gasodutos e oleodutos, visando proteger todo o sistema da deterioração que pode provocar perdas econômicas, diminuição da eficiência de processos, contaminação de produtos, acidentes ambientais e riscos à vida humana. Em geral, os inibidores de corrosão são utilizados juntamente com biocidas e surfactantes em misturas complexas contendo dois ou mais componentes inibidores, como sais de amônio quaternário, amidas, imidazolinas, éster de fosfato e outros. ${ }^{5,6}$

Os sais de amônio quaternário são uma classe de compostos de interesse particular, pois atuam como surfactantes, inibidores de corrosão e biocidas. São um dos tipos de inibidores de corrosão mais utilizados, tanto na produção de óleo, quanto na produção de gás. A solubilidade em água, associada à baixa biodegrabilidade destas classes de inibidores de corrosão, confere um risco potencial de

\footnotetext{
*e-mail: herllaine.almeida@inea.rj.gov.br
}

bioacumulação em ambientes marinhos. A presença de inibidores de corrosão na água produzida pode contribuir significativamente para contaminação da fauna e da flora, comprometendo a alimentação de procedência marinha, provocando um impacto visual negativo e a perda temporária do uso de áreas qualificadas como pesqueiras, de recreação e lazer. ${ }^{7}$

Determinar com precisão a concentração de sais de amônio quaternário em água é importante para atender os níveis admitidos na água produzida a ser descartada, visto que estas substâncias impactam o meio ambiente..$^{8-11}$

A escolha da técnica analítica para monitorar os sais de amônio quaternário na água produzida depende da exatidão, velocidade, sensibilidade e seletividade desejadas. Das técnicas aplicadas a este tipo de análise, a fluorescência é um método seletivo, pois nem todas as moléculas exibem fotoluminescência. $\mathrm{O}$ fenômeno da fluorescência é o processo inverso da absorção de radiação eletromagnética e é produzido pela emissão de um fóton a partir do retorno do estado eletrônico excitado para o fundamental de moléculas.

Nem todas as moléculas fluorescem. Teoricamente é difícil predizer se uma molécula exibirá fotoluminescência, mas é possível observar certas características gerais na sua estrutura que favoreçam o processo de desativação radiativo eficiente. A maioria dos compostos que fluorescem são aromáticos e alguns poucos compostos alifáticos, alicíclicos carbonilados ou estruturas com ligações duplas altamente conjugadas também podem exibir fluorescência. Isto se deve ao fato dos compostos aromáticos terem níveis de transição $\left(\pi \rightarrow \pi^{*}\right)$ de baixa energia. Já no caso de substâncias com estruturas alifáticas, a energia necessária para promover transições $\sigma \rightarrow \sigma^{*}$ é tão alta que ocasiona decomposição da molécula. A rigidez na estrutura molecular restringe a liberdade vibracional, minimizando significativamente os relaxamentos vibracionais, aumentando a fluorescência. ${ }^{12}$

O presente trabalho teve como objetivo principal estudar a aplicabilidade da técnica de emissão por fluorescência de ultravioleta na quantificação de soluções aquosas de um inibidor de corrosão, que tem como matéria ativa um sal alquil-aril de amônio quaternário. Além dessa quantificação, também se pretendeu avaliar a interferência de 
alguns componentes que geralmente estão presentes na matriz de água produzida.

\section{PARTE EXPERIMENTAL}

Os padrões de inibidor de corrosão utilizados, o cloreto de 2-metil-4-dodecil-benzil-trimetil amônio (MDBTAC) e cloreto de dodecil-trimetil amônio (DTAC) foram fornecidos pela Chem Service INC. O inibidor de corrosão comercial (ICC) avaliado foi o Cortron JRN 365, obtido da Champion Technologies do Brasil. A matéria ativa do produto comercial é um sal de amônio quaternário 10 a $30 \%$ em massa. O biocida Tolcide PS75 ou THPS75 (sulfato de tetra-hidroximetilfosfônico) solução $75 \%$ em massa foi obtido da Rhodia (Inglaterra).

As análises espectroscópicas de absorbância na região do UV-Vis foram realizadas em espectrofotômetro modelo HP 8453, da Hewlett Packard. As análises espectroscópicas de fluorescência na região do UV-Vis foram realizadas em espectrofluorímetro modelo LS55, da Perkin-Elmer. Nos dois equipamentos empregaram-se cubetas de quartzo de caminho ótico de $1,0 \mathrm{~cm}$ e água destilada como solução de referência.

\section{RESULTADOS E DISCUSSÃO}

\section{Determinação do comprimento de onda de excitação ideal}

O comprimento de onda de excitação ideal para registrar o espectro de emissão de um composto fluorescente é, em geral, escolhido em função de seu espectro de absorção. Na maior parte dos casos, este comprimento de onda corresponde ao máximo de absorção e fornece um elevado sinal fluorescente. ${ }^{13}$

Foram obtidos espectros de absorbância na região do UV-Vis de soluções aquosas de 250; 100 e 30,0 mg kg-1 do MDBTAC, do DTAC e do ICC. Para o MDBTAC observou-se forte absorção nos comprimentos de onda 205, 225 e $270 \mathrm{~nm}$ (Figura 1S, material suplementar), valores estes muito próximos aos registrados para compostos com anel benzênico monossubstituído. ${ }^{14}$

Para o DTAC, foram observadas bandas fracas, com máximo de absorção localizado abaixo de $200 \mathrm{~nm}$ (Figura 2S, material suplementar). O DTAC é um composto formado por ligações simples. A excitação destas substâncias envolve a promoção de elétrons de orbitais $\sigma \rightarrow \sigma^{*}$, e esta transição necessita de grande energia (abaixo de $200 \mathrm{~nm}$ ), correspondendo, no vácuo, à energia da região do ultravioleta. Além disso, os valores de absorbância verificados são muito baixos (de 0 a 0,156 ) e possuem baixa relação sinal ruído quando comparados com os espectros obtidos para o MDBTAC.

Para o ICC observou-se duas regiões de máximo de absorção em torno de 205 e $235 \mathrm{~nm}$ (Figura 3S, material suplementar). Estes valores, apesar de serem próximos aos característicos de anéis aromáticos monossubstituídos, também podem ter sido afetados em função da estrutura da molécula cromófora, do solvente da solução e do efeito da conjugação com outros cromóforos e auxócromos presentes na composição da solução comercial, tais como compostos contendo o grupo amino, que absorvem em torno de $215 \mathrm{~nm}$ e o etanol, que apresenta absorção em $184 \mathrm{~nm} .^{14}$

A análise comparativa dos máximos de absorção mostra que os valores de absorbância são maiores em comprimentos de onda menores que $240 \mathrm{~nm}$. Entretanto, valores próximos aos extremos da radiação emitida, pela fonte do equipamento, podem provocar desvios instrumentais como, por exemplo, a geração de falsos picos de absorção devido ao espalhamento da luz. O espectrofotômetro de UV utilizado opera com uma combinação de lâmpadas: uma de deutério para a região do UV e uma de tungstênio para o visível; esta combinação oferece ao equipamento uma faixa de resposta espectral entre 190 e $1100 \mathrm{~nm}$. Dessa forma, no presente estudo escolheu-se o comprimento de onda de $230 \mathrm{~nm}$ para excitação nos experimentos de fluorescência. ${ }^{14}$

\section{Obtenção do espectro de emissão por fluorescência de ultravioleta}

As análises espectroscópicas de fluorescência na região do UVVis foram realizadas nas amostras de soluções aquosas de aproximadamente $10 \mathrm{mg} \mathrm{kg}^{-1}$ do MDBTAC, do ICC e do DTAC, tendo a água destilada como referência. Os resultados encontram-se na Figura 1. As semelhanças entre os espectros do ICC e do MDBTAC em solução aquosa sugerem que ambos possuem os mesmos componentes fluorescentes. As diferenças nas intensidades e nos alargamentos das curvas de fluorescências podem indicar que o sal trialquil-aril quaternário de amônio possui detalhes na estrutura molecular, como número de átomos de carbono e a posição do anel benzênico, diferentes do padrão (MDBTAC). Além disso, estas diferenças podem ser explicadas, também, pelo fato do ICC ser uma mistura de produtos químicos, apresentando então uma composição distinta do MDBTAC.

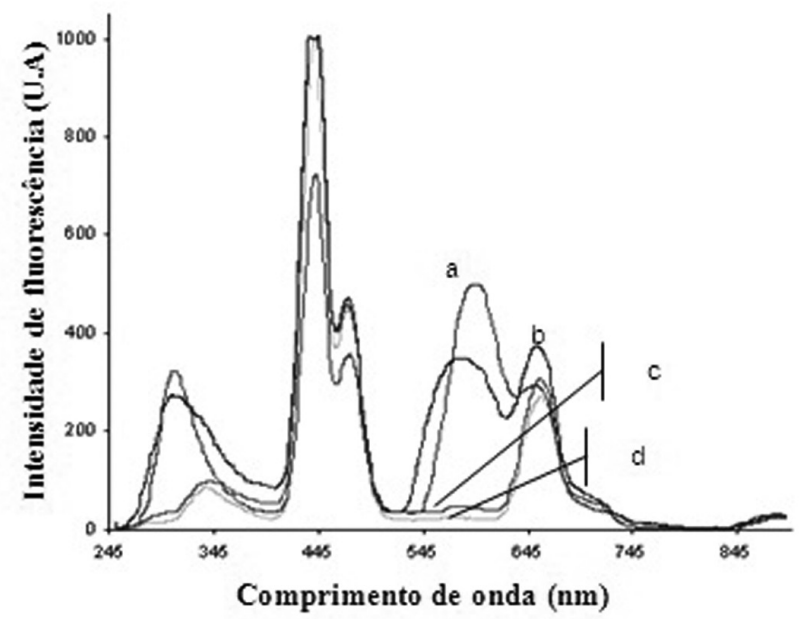

Figura 1. Espectros de emissão $\left(\lambda_{e x}=230 \mathrm{~nm}\right)$ de solução aquosa: (a) 10,02 mg kg-1 de MDBTAC, (b) 10,28 mg kg-1 de ICC, (c) 10,01 $\mathrm{mg} \mathrm{kg}^{-1}$ de DTAC e (d) água

Através da análise comparativa foi possível identificar 5 regiões distintas em torno dos seguintes comprimentos de onda: 306, 346, 442, 475, 593 e $660 \mathrm{~nm}$. Em 306 e $593 \mathrm{~nm}$ podem ser identificados máximos de emissão, tanto no ICC, quanto no MDBTAC, característicos da presença de um hidrocarboneto aromático com um anel aromático na composição do ICC e a formação de dímeros excitados, chamados excímeros (E), em torno de $593 \mathrm{~nm}$. A formação de excímeros e sua fluorescência foram identificadas inicialmente para soluções de pireno, que apresenta uma banda fluorescente em torno de 400 nm. Em soluções de concentração superior a $0,001 \mathrm{~mol} \mathrm{~L}^{-1}$ ocorre o aparecimento de uma banda em torno de $500 \mathrm{~nm}$. Atualmente, já se sabe que isto é comum na maioria dos hidrocarbonetos aromáticos e seus derivados, e que esta banda pode ser utilizada para caracterizações e quantificações. A banda fluorescente correspondente a um excímero é localizada em comprimentos de onda maiores do que os do monômero. Além disso, quando se aumenta a concentração não ocorrem mudanças no espectro de absorção da solução correspondente ao excímero, o que mostra que sua formação ocorre somente no estado excitado, pois o excímero não absorve luz. ${ }^{15,16}$

As emissões verificadas nos outros comprimentos de onda são perturbações que podem ser atribuídas a fenômenos de difusão da 
luz, como o espalhamento Rayleigh e Raman, conforme evidenciado por alguns autores em estudos de fluorescência de águas naturais contendo matéria orgânica dissolvida. A difusão Rayleigh é provocada pela presença, sobre o trajeto do raio luminoso incidente, de "partículas" de tamanho muito inferior ao comprimento de onda da luz incidente, como as moléculas de água. No espectro de emissão da água seriam esperadas bandas intensas de espalhamento em 230, 460, 690 e $920 \mathrm{~nm}$ (respectivamente, $1^{\mathrm{a}}, 2^{\mathrm{a}}, 3^{\mathrm{a}}$ e $4^{\mathrm{a}}$ ordem), tendo sido registradas, tanto na água, quanto nas soluções de estudo, em 442,475 e $660 \mathrm{~nm}^{13,17}$

A emissão verificada em torno de $346 \mathrm{~nm}$ pode ser qualificada como difusão ou dispersão Raman. A posição relativa da banda Raman depende da natureza das moléculas do solvente utilizado e, da mesma forma que a difusão Rayleigh, também pode se manifestar em $2^{\mathrm{a}}, 3^{\mathrm{a}}$ e $4^{\mathrm{a}}$ ordem. Este espalhamento é um fenômeno raro, que exige altas concentrações da espécie provocadora de espalhamento ou uma fonte de luz de alta energia (como um laser), que também pode ser verificado neste tipo de estudo, pois, novamente, a água é a única molécula em concentração suficiente para provocar bandas fracas, mas visíveis de espalhamento Raman a partir de medidas de fluorescência. ${ }^{13,17-20}$

\section{Determinação de parâmetros instrumentais de emissão de fluorescência do ICC}

Para avaliar as influências de variáveis de parâmetros instrumentais sobre a emissão de fluorescência do ICC, foram estudadas as larguras das fendas de excitação e emissão e a velocidade de varredura para obtenção dos espectros. A escolha de testar algumas variáveis de parâmetros instrumentais diretamente sobre o produto comercial visou contemplar todas as variabilidades presentes na amostra, o que não estaria presente em uma amostra padrão de um sal de amônio quaternário.

Em um primeiro conjunto de experimentos, avaliaram-se as medidas de fluorescência de solução de ICC com concentração aproximada de $10 \mathrm{mg} \mathrm{kg}^{-1}$, inicialmente, variando-se a largura das fendas de excitação e de emissão de 2,5 a $15 \mathrm{~nm}$ (mantendo-se a velocidade de varredura padrão de $100 \mathrm{~nm}$ min $^{-1}$ ). As larguras das fendas de excitação e emissão são variáveis que interferem diretamente na intensidade do sinal do fluorófilo.

O sinal fluorescente é proporcional ao quadrado do produto das larguras das fendas de excitação e de emissão (QPLF), e o uso de larguras estreitas das fendas resulta em um aumento da seletividade que, em geral, resulta em um decréscimo na sensibilidade, enquanto que maiores larguras das fendas produzem o efeito oposto. ${ }^{21,22}$

Os resultados apresentados na Tabela 1 indicaram que nas combinações C2, C3 e C5, à medida que se aumentou o tamanho da fenda, o sinal fluorescente também cresceu. Em função do baixo sinal fluorescente, elevado ruído e da sobreposição de bandas apresentadas durante a obtenção dos espectros, descartou-se a utilização das combinações dos experimentos $\mathrm{C} 1$ e $\mathrm{C} 4$.

Dois efeitos mencionados na literatura ${ }^{21,22} \mathrm{e}$ indicados a seguir foram considerados na escolha das melhores larguras de fenda. $\mathrm{O}$ primeiro consiste no uso de fendas de excitação menores, o que promove a incidência de radiação em uma faixa mais estreita de comprimento de onda e, consequentemente, mais próxima do máximo de excitação da amostra, aumentando-se dessa maneira o rendimento quântico da fluorescência, ou seja, a razão entre o número de fótons emitidos por fluorescência e o número de fótons absorvidos. O segundo efeito decorre do fato que o uso da fenda de emissão totalmente aberta pode ocasionar a geração de radiações espúrias, que provocam interferências espectrais, que podem diminuir a intensidade do sinal fluorescente. Para garantir a melhor relação entre seletividade e
Tabela 1. Intensidade do sinal fluorescente de solução aquosa $9,96 \mathrm{mg} \mathrm{kg}^{-1}$ do ICC $\left(\lambda_{\text {ex }} 230 \mathrm{~nm}\right)$ utilizando diferentes combinações de largura de fenda de excitação e emissão

\begin{tabular}{|c|c|c|c|c|c|}
\hline \multirow[b]{2}{*}{$\mathrm{C}^{\mathrm{a}}$} & \multicolumn{2}{|c|}{$\mathrm{LF}^{\mathrm{b}}(\mathrm{nm})$} & \multirow{2}{*}{$\begin{array}{l}\mathrm{QPLF}^{\mathrm{d}} \\
\left(\mathrm{nm}^{4}\right)\end{array}$} & \multicolumn{2}{|c|}{$\mathrm{IF}^{\mathrm{e}}$ (U.A.) } \\
\hline & $E X^{c}$ & $\mathrm{EM}^{\mathrm{d}}$ & & $\begin{array}{c}\lambda_{1}{ }^{\mathrm{f}} \\
306 \mathrm{~nm}\end{array}$ & $\begin{array}{c}\lambda_{2}{ }^{\mathrm{f}} \\
593 \mathrm{~nm}\end{array}$ \\
\hline 1 & 2,5 & 2,5 & 39,0625 & 15,85 & 22,76 \\
\hline 2 & 10 & 10 & 10000 & 189,2 & 259,3 \\
\hline 3 & 10 & 15 & 22500 & 413,3 & 560,3 \\
\hline 4 & 15 & 10 & 22500 & 55,30 & 78,94 \\
\hline 5 & 15 & 15 & 50625 & 134,1 & 176,2 \\
\hline
\end{tabular}

a) Combinações; b) largura de fenda; c) excitação; d) emissão; e) intensidade de fluorescência; f) comprimento de onda

sensibilidade nas análises seguintes, definiu-se a fenda de excitação em $10 \mathrm{~nm}$ e a fenda de emissão em $15 \mathrm{~nm}$.

Em um segundo experimento, foram obtidos espectros de emissão com uma solução de $10,54 \mathrm{mg} \mathrm{kg}^{-1}$ de inibidor de corrosão, variando-se a velocidade de varredura entre 10 e $500 \mathrm{~nm} \mathrm{~min}^{-1}$. Constatou-se que a intensidade de fluorescência diminuiu à medida que se reduziu a velocidade de varredura aplicada (variação de 500 a $10 \mathrm{~nm} \mathrm{~min}{ }^{-1}$ ). No comprimento de onda de $306 \mathrm{~nm}$, o sinal fluorescente decresceu de 405, 62 para 388,05 U.A; em 593 nm decresceu de 554,92 para 505,98 U.A. O decréscimo do sinal fluorescente pode ser explicado pela fotodegradação do ICC; alguns trabalhos têm demonstrado a ocorrência deste tipo de degradação induzida (por fontes emissoras de luz). ${ }^{23}$

A volatilização do ICC também pode explicar tal decréscimo do sinal fluorescente, devido ao maior tempo de radiação incidente, além da formação de filme adsorvente no frasco da amostra. Nesse sentido, nos próximos experimentos, manteve-se a velocidade de varredura de $100 \mathrm{~nm} \mathrm{~min}^{-1}$ com o intuito de obter espectros de boa resolução, num tempo viável de análise (1,9 min) e sem minoração do sinal fluorescente do fluorófilo.

\section{Estabilidade do ICC em função do tempo de estocagem}

Os compostos quaternários de amônio têm tendência em aderir na superfície dos frascos de armazenamento de amostra, tanto plásticos quanto vidros, levando a resultados de análise imprecisos. ${ }^{24}$ Para avaliar a estabilidade da solução em função do tempo de armazenagem e prever futuras orientações quanto à amostragem foram realizados experimentos com solução de ICC na concentração de $10 \mathrm{mg} \mathrm{kg}^{-1}$ em diferentes solventes: água, água/isopropanol (10:1 e 1:1) e água/ metanol (1:10 e 1:1). Também foi avaliada a influência da temperatura, com armazenamento a $6,6^{\circ} \mathrm{C}$, sendo obtidos espectros após retornar à temperatura ambiente $\left(20 \mathrm{a} 24^{\circ} \mathrm{C}\right)$. Em todos os experimentos a intensidade do sinal fluorescente selecionado para análise comparativa foi no comprimento de onda de $306 \mathrm{~nm}$ e a intensidade do sinal fluorescente foi avaliada em um intervalo de 3 dias.

Observou-se, na solução sem preservação, a diminuição significativa do sinal fluorescente de 358,74 para 201,39 U.A., uma redução de cerca de $40 \%$. A formação do filme sobre os frascos de armazenamento pode reduzir a concentração em fase aquosa da espécie em estudo disponível em solução com o tempo, diminuindo a intensidade do sinal fluorescente, necessitando então que as soluções preparadas em laboratório ou as amostras de água produzidas obtidas sejam analisadas imediatamente.

Os processos de formação do filme adsorvente e da diminuição do sinal fluorescente podem ser melhor compreendidos pelo estudo 
da característica surfactante ou tensoativa que os sais de amônio quaternário apresentam. Tais compostos se caracterizam por possuir duas regiões distintas na mesma molécula: uma região polar (hidrofílica) e outra região não polar (hidrofóbica). ${ }^{25}$

Os sais de amônio quaternário possuem atividade na superfície entre duas fases, tais como ar-água, óleo-água, e na superfície. Através da formação de agregados moleculares, estes surfactantes geram filmes não permeáveis que funcionam como uma barreira entre a superfície de um metal que se pretende proteger da corrosão, por exemplo, e o fluido presente no ambiente, devido à ligação entre o par de elétrons livres do nitrogênio e o metal. O mesmo tipo de interação também pode ser verificada nos frascos de armazenamento ou nas cubetas de análise. ${ }^{26,27}$

Estes agregados moleculares, chamados de micelas, de forma dinâmica se associam espontaneamente em solução aquosa a partir de certa concentração crítica (CMC), formando grandes agregados moleculares de dimensões coloidais. ${ }^{28} \mathrm{~A}$ estabilidade de agregados do tipo monocapa, como os que se formam a partir de sais de amônio quaternário, como o MDBTAC e o presente no ICC, pode ser comprometida em horas ou dias. Assim, soluções preparadas recentemente apresentam intensidade de fluorescência maior devido à existência destes agregados, enquanto que as leituras nos dias seguintes diminuem pela perda da característica micelar destas soluções.

A diminuição do sinal fluorescente é atribuída à formação e destruição dos agregados micelares formados pelo ICC em função do tempo, o que acarreta mudança de polaridade afetando, assim, a energia relativa do estado excitado do fluorófilo, que no caso do ICC é o anel benzênico presente em sua estrutura molecular. ${ }^{29}$ Diversos autores $^{30,31}$ evidenciaram a influência de ambientes micelares em estudos de cinéticas de reações, determinação da CMC, aplicação de sondas fluorescentes, dentre outros.

Para verificar a capacidade de solventes alcoólicos em manter o inibidor de corrosão disperso na água, foram preparadas misturas com metanol e isopropanol. Solventes polares nos quais o inibidor de corrosão é prontamente solúvel são comumente utilizados na lavagem das vidrarias de trabalho. ${ }^{32}$ A diminuição do sinal fluorescente na solução contendo $10 \%$ de metanol foi de 386,37 para 194,85 U.A., uma queda de $50 \%$ no sinal. Já na solução contendo $10 \%$ de isopropanol foi 376,48 para 285,84 U.A., uma redução de $24 \%$ (Figuras 4S e 5S, material suplementar).

Ainda avaliando a adição de solventes, nas soluções de $50 \%$ em massa de alcoóis ocorreu a extrapolação da escala (Figuras 6S e 7S, material suplementar) não sendo possível calcular a variação da intensidade do sinal fluorescente. A influência do solvente no espectro eletrônico ocorre devido as suas propriedades eletrostáticas. Uma mudança da polaridade do solvente ou de seu caráter prótico afeta a energia relativa do estado excitado. ${ }^{29}$

Também se verificou que a solução de ICC mantida sob refrigeração apresentou uma queda de $35 \%$ do sinal fluorescente, de 372,56 para 198,12 U.A., sendo este valor 5\% menor do que para a amostra não refrigerada. Isso demonstrou que a refrigeração da solução de inibidor de corrosão e o controle do ambiente da sala em baixas temperaturas são determinantes para a reprodutibilidade das medidas de fluorescência, pois diminuem os efeitos da desativação por conversão externa do fluorófilo. ${ }^{14,16,32}$. Assim, constatou-se que a adição de isopropanol à solução foi o método mais eficiente em manter o inibidor de corrosão disperso.

\section{Determinação de curva de calibração do MDBTAC e do ICC}

Para o MDBTAC, conforme pode se verificar na Figura 2, a adição de concentrações superiores a $5,19 \mathrm{mg} \mathrm{kg}^{-1}$ produziu diminuição significativa da IF da solução, devido ao fenômeno de autossupressão, no qual ocorre a redução do rendimento quântico de um sistema luminescente, devido à interação do fluorófilo com determinadas substâncias denominadas de supressoras ou, então, devido ao elevado nível de concentração das espécies. As moléculas excitadas transferem energia às moléculas não excitadas vizinhas, diminuindo assim a intensidade das bandas fluorescentes.$^{33}$ Após a exclusão dos valores de concentração superiores a $5,19 \mathrm{mg} \mathrm{kg}^{-1}$, para cada um dos comprimentos de onda testados, foram obtidas as seguintes equações:

$$
\begin{array}{ll}
\mathrm{y}_{(306)}=46,3 \mathrm{x}+48,2 & \mathrm{R}^{2}=0,9891 \\
\mathrm{y}_{(593)}=74,7 \mathrm{x}+78,8 & \mathrm{R}^{2}=0,9741
\end{array}
$$

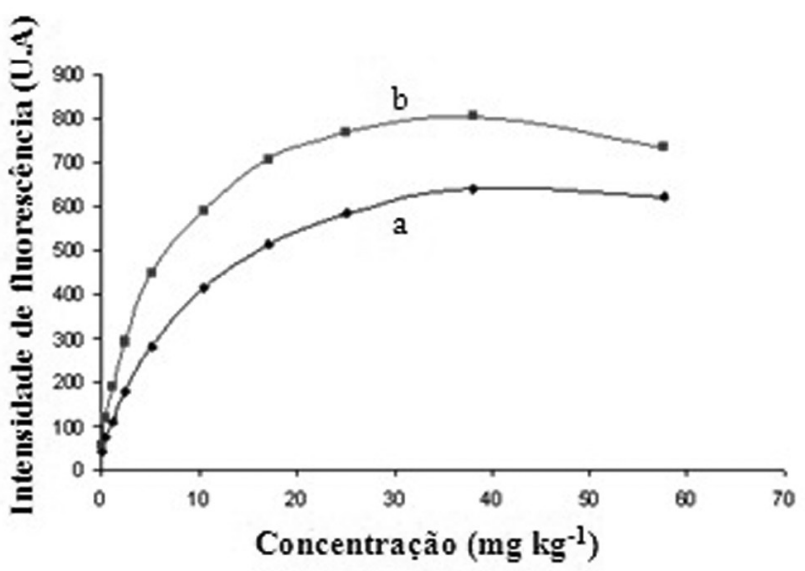

Figura 2. Variação da IFC $\left(\lambda_{e x}=230 \mathrm{~nm}\right)$ de solução aquosa concentrada de MDBTAC em (a) $306 \mathrm{~nm}$ e (b) $593 \mathrm{~nm}$

Com base na análise dos resultados para o ICC (Figura 3) verificou-se que concentrações superiores a 19,42 mg kg-1 acarretaram a autossupressão da IF da solução de ICC. Para o intervalo de concentrações de 0,136 a 19,42 $\mathrm{mg} \mathrm{kg}^{-1}$ foram obtidas as seguintes equações:

$$
\begin{array}{cc}
\mathrm{y}_{(306)}=20,7 \mathrm{x}+96,1 & \mathrm{R}^{2}=0,9959 \\
\mathrm{y}_{(593)}=25,0 \mathrm{x}+123,6 & \mathrm{R}^{2}=0,9906
\end{array}
$$

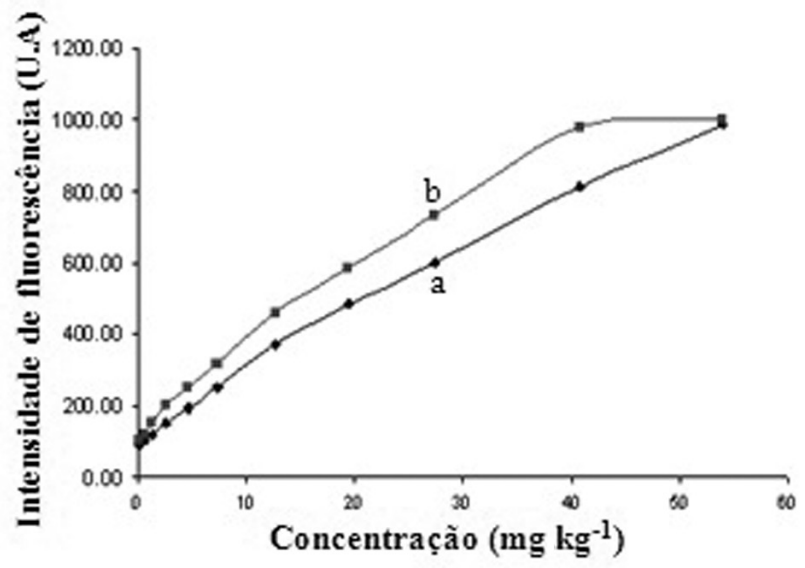

Figura 3. Variação da IFC $\left(\lambda_{e x}=230 \mathrm{~nm}\right)$ de solução aquosa concentrada de ICC em (a) $306 \mathrm{~nm}$ e (b) $593 \mathrm{~nm}$

Os coeficientes de correlação dessas equações indicam a linearidade do método, tanto para o MDBTAC, quanto para o ICC, nos intervalos de concentração de 0,133 a 5,19 e 0,136 a 19,42 $\mathrm{mg} \mathrm{kg}^{-1}$, respectivamente. As diferenças observadas nos intervalos de concentração das curvas são devidas ao efeito da supressão do sinal fluorescente por aumento de concentração ser menos pronunciado no ICC que no padrão puro de 
MDBTAC. O produto comercial apresenta o sal de amônio quaternário, em uma faixa de concentração de 10 a $30 \%$ em massa e as soluções foram preparadas levando-se em conta a mistura, ou seja, $100 \%$, e não o teor do componente surfactante (ingrediente).

Outro fator relevante é a presença na formulação comercial (ICC) de uma mistura de substâncias tais como etanol, isopropanol, xileno, etilbenzeno, dentre outras. A combinação destas substâncias pode interferir nos espectros de emissão fluorescente, seja por pequenos deslocamentos do comprimento de onda de máxima emissão, quanto por alteração da intensidade de fluorescência. ${ }^{33}$

\section{Estudo de interferentes}

Para avaliar a influência de possíveis interferentes sobre o sinal fluorescente de soluções aquosas de ICC, foram realizados estudos com os três principais componentes da água produzida: sais dissolvidos, biocida e hidrocarbonetos aromáticos (benzeno, tolueno, etilbenzeno e xileno).

\section{Influência da salinidade}

Foi investigado o efeito da salinidade do meio aquoso, pois a solubilidade do inibidor de corrosão normalmente diminui com o aumento da concentração de sais. ${ }^{27}$ Para o estudo da influência da salinidade, foram preparadas 4 soluções salinas em diferentes concentrações baseadas em águas produzidas do Mar do Norte e de alguns campos do Brasil, conforme apresentado na Tabela 2.

Tabela 2. Composição de águas salinas preparadas baseadas em águas produzidas do Mar do Norte e de alguns campos do Brasil

\begin{tabular}{ccccc}
\hline & \multicolumn{4}{c}{$\operatorname{Comp}^{\mathrm{b}}\left(\mathrm{mg} \mathrm{kg}^{-1}\right)$} \\
\cline { 2 - 4 } $\mathrm{Carac}^{\mathrm{a}}$ & \multicolumn{3}{c}{$\mathrm{CB}^{\mathrm{f}}$} \\
\cline { 2 - 4 } & $\mathrm{BS}^{\mathrm{c}}$ & $\mathrm{MS}^{\mathrm{d}}$ & $\mathrm{AS}^{\mathrm{e}}$ & \\
\hline $\mathrm{Cl}^{-}$ & 52055 & 36389 & 122784 & 73975 \\
$\mathrm{Ca}^{2+}$ & 1918 & 6150 & 13118 & 8210 \\
$\mathrm{Mg}^{2+}$ & 1312 & 1415 & 16222 & 429 \\
$\mathrm{Ba}^{2+}$ & 13,12 & -- & 14,90 & 26,20 \\
$\mathrm{Na}^{+}$ & 29069 & 13865 & 33878 & 37442 \\
$\mathrm{~K}^{+}$ & -- & -- & -- & 492 \\
$\mathrm{SO}_{4}^{2-}$ & -- & -- & -- & 84,40 \\
$\mathrm{HCO}_{3}^{-}$ & -- & -- & -- & 292 \\
$\mathrm{pH}^{-}$ & 5 & 6 & 5 & 6 \\
\hline
\end{tabular}

a) Característica; b) composição em água destilada; c) baixa salinidade; d) média salinidade; e) alta salinidade; f) campos do Brasil

As soluções salinas preparadas, baseadas na composição de águas produzidas de alta (A), média (M) e baixa (B) salinidade, apresentaram leve turvação e quando observadas sob uma lupa com capacidade de aumento de 40x apresentavam material em suspensão. A água sintética preparada a partir da composição típica de uma água produzida de campos brasileiros (CB) apresentou turvação acentuada perceptível a olho nu. Esta turvação se deve à presença dos sais em suspensão, que provocam perturbações óticas nos espectros, características do espalhamento da luz, do tipo Rayleigh e do tipo Tyndall, que é causado pela reflexão da luz por partículas coloidais ou em suspensão e que não são observadas ao se analisar a água destilada, que podem interferir ou ser confundidas com a fluorescência da amostra. ${ }^{13,19}$ Para avaliar o efeito da turvação, uma fração dessas soluções foi filtrada a vácuo, com filtro de acetato de celulose com diâmetro nominal de poro de $0,45 \mu \mathrm{m}$.
Os gráficos das Figuras 4 e 5 apresentam as intensidades de emissão fluorescente em 306 e 593 nm, verificadas nas soluções preparadas. Em ambos os comprimentos de onda, as IFs das soluções não filtradas são maiores do que as verificadas nas soluções filtradas. O espalhamento provoca extrapolação da escala nas soluções não filtradas, exceto nas soluções de média salinidade não filtradas, que registraram valores menos intensos. Este fenômeno pode ser atribuído à ausência de sais de bário na composição da solução. ${ }^{34}$ Os sais de bário tendem a ser menos solúveis e formar suspensões. Assim, soluções que não apresentem estes sais provocam menor espalhamento, como foi verificado em todas as soluções de média salinidade filtradas ou não, com e sem inibidor de corrosão.

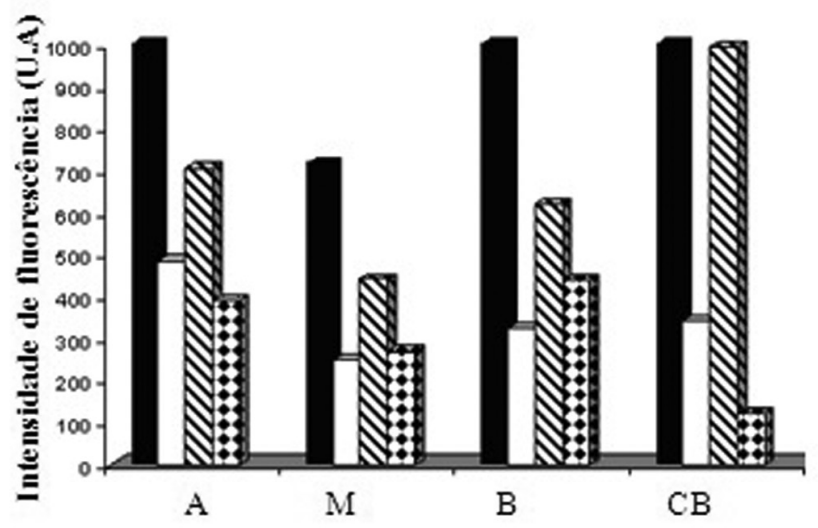

Figura 4. Variação da IF em 306 nm de soluções salinas com e sem inibidor de corrosão e submetidas ou não à filtração: sem filtrar isenta de IC (ロ), filtrado isenta de IC ( $\square)$, sem filtrar com IC $(\mathbb{N})$, filtrado com IC (国), alta salinidade (A), média salinidade $(M)$, baixa salinidade $(B)$ e campos brasileiros $(C B)$

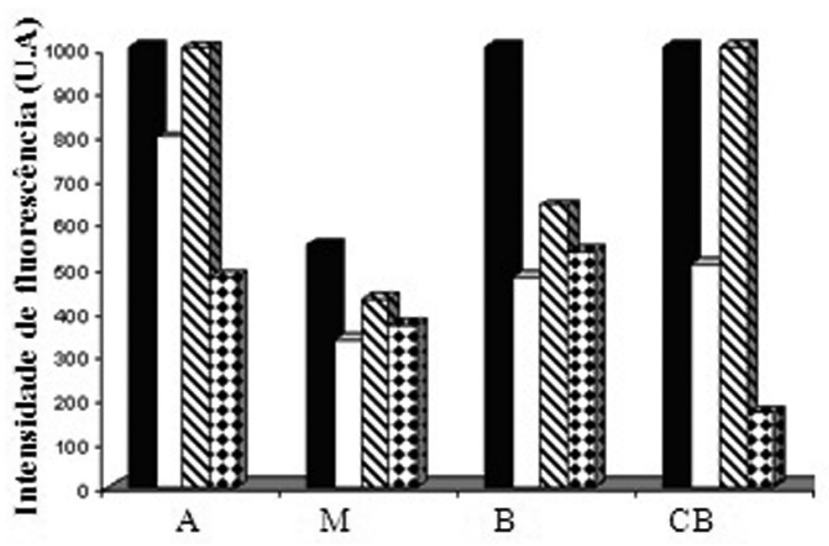

Figura 5. Variação da IF em 593 nm de soluções salinas com e sem inibidor de corrosão e submetidas ou não à filtração: sem filtrar isenta de IC (ロ), filtrado isenta de IC $(\square)$, sem filtrar com IC $(\mathbb{\mathbb { N }})$, filtrado com IC (国), alta salinidade (A), média salinidade (M), baixa salinidade (B) e campos brasileiros (CB)

Comparando-se as soluções sem filtrar, sem filtrar com inibidor e filtrada com inibidor, verifica-se que soluções contendo o inibidor de corrosão possuem valores de IF menores. O esperado seria que as soluções contendo a espécie fluorescente apresentassem bandas mais intensas do que as isentas de inibidor de corrosão, devido a sua propriedade fluorescente intrínseca. Este comportamento anômalo pode ser explicado pela característica surfactante do inibidor de corrosão que possui a ação de dissolver sais, como os de cálcio e magnésio, impedindo assim que formem aglomerados. Quanto menos aglomerados de sais são formados, menor o tamanho das partículas em solução, diminuindo assim o efeito Tyndall, que intensifica as 
bandas de emissão, incorrendo em erros nas medidas..$^{13,34,35}$

\section{Influência da presença de biocidas}

A partir de espectros de fluorescência de ultravioleta de soluções de diferentes concentrações de biocida (sulfato de tetra-hidroximetilfosfônico) em água destilada (Figura 8S, material suplementar), excitadas e observadas nos mesmos comprimentos de onda de estudo do inibidor de corrosão, pode-se concluir que as bandas observadas são espalhamentos Raman e Rayleigh e não foram verificadas emissões significativas em 306 e $593 \mathrm{~nm}$. As intensidades de fluorescência registradas foram similares às do líquido de referência, água destilada, exceto nas soluções de 948,2 e 1939,5 mg kg-1 em $306 \mathrm{~nm}$. Estas soluções são as de maior concentração de biocida e, então, ocorre a intensificação das bandas de espalhamento, devido à saturação destas soluções.

Muitas substâncias podem funcionar como supressoras e por diversos mecanismos de interação molecular. ${ }^{33}$ Constatou-se que o biocida provocou a supressão do sinal fluorescente do inibidor de corrosão. Para avaliar se a supressão era devida unicamente à presença do biocida ou à supressão intrínseca do inibidor de corrosão em água, foram analisadas soluções com concentração variável de biocida. Os resultados dos espectros (Figura 9S, material suplementar) revelaram que com cerca de $2000 \mathrm{mg} \mathrm{kg}^{-1}$ do biocida, concentração utilizada em alguns campos de produção do Brasil, o sinal fluorescente é significantemente reduzido. Em concentrações intermediárias, em torno de 1000 e $500 \mathrm{mg} \mathrm{kg}^{-1}$, observa-se a diminuição deste efeito supressor do biocida. Assim, indica-se a diluição da amostra em pelo menos quatro vezes para que o teor de biocida seja inferior a $500 \mathrm{mg} \mathrm{kg}^{-1}$.

\section{Influência da presença de BTEX}

Foram obtidos espectros de emissão e medidas de fluorescência, sob as mesmas condições aplicadas ao ICC, de soluções aquosas de benzeno, tolueno, etilbenzeno e xileno (BTEX) em diferentes concentrações. Devido à solubilidade dos BTEX em água, inclusive em água do mar, as semelhanças em termos de fórmula estrutural, e suas emissões fluorescentes em regiões próximas às do estudo do inibidor de corrosão, a presença de compostos aromáticos na água produzida interfere significantemente na quantificação destas substâncias (Figura 10S, material suplementar). Estes fluorófilos emitem em torno dos mesmos comprimentos de onda do inibidor de corrosão, seus máximos de emissão foram em 293 e 572 nm, quando excitados sob as mesmas condições. A emissão característica do benzeno e tolueno em álcool, registrada em espectros encontrados em referências bibliográficas, está entre 270 e $310 \mathrm{~nm}$, valores estes próximos dos resultados obtidos nos ensaios com BTEX. Todas as outras bandas verificadas são denominadas espalhamento característico da água. ${ }^{16,36}$

\section{CONCLUSÕES}

Soluções aquosas preparadas com o padrão de um sal trialquil-aril quaternário de amônio e o inibidor de corrosão apresentaram máximos de emissão fluorescente no ultravioleta, comuns em 306 e 593 nm.

Os estudos preliminares de variáveis instrumentais definiram os melhores parâmetros para obtenção de espectros bem resolvidos e detectáveis em um tempo otimizado, cerca de 2 min: largura de fendas de 10 e $15 \mathrm{~nm}$, respectivamente, para excitação e emissão e uma velocidade de varredura de $10 \mathrm{~nm} \mathrm{~min}^{-1}$.

As curvas de calibração obtidas em água destilada mostraram que a faixa determinável de MBCTA é de 0,133 a 5,19 $\mathrm{mg} \mathrm{kg}^{-1}$ e para o inibidor de corrosão de 0,136 a $19,42 \mathrm{mg} \mathrm{kg}^{-1}$. Valores de maior concentração apresentaram efeito da supressão do sinal fluorescente.

Ao se avaliar a estabilidade da solução de inibidor de corrosão, constatou-se que o isopropanol é o mais eficiente em manter a intensidade do sinal fluorescente, frente à água, o etanol e a refrigeração.

A presença de sais na água provoca perturbações nos espectros de emissão do inibidor de corrosão, devido aos fenômenos de espalhamento Rayleigh e Tyndall. A filtração prévia da amostra a ser analisada, então, é o procedimento para minimização deste interferente.

O fenômeno de supressão também foi evidenciado quando estudada a interferência da presença de biocida sobre a fluorescência do inibidor de corrosão. A diluição da solução é indicada para evitar a diminuição do sinal fluorescente.

Compostos aromáticos, por apresentarem máximos de emissão próximos das soluções de estudo, em torno de 295 e $572 \mathrm{~nm}$, e semelhanças nas estruturas moleculares, afetam significantemente o sinal fluorescente.

A fluorescência de ultravioleta traz uma possibilidade de quantificação em água de um inibidor de corrosão do tipo sal de amônio quaternário, uma substância utilizada em larga escala e que apresenta elevada toxicidade para o meio ambiente. Entretanto, os efeitos da matriz água produzida como perturbações óticas, supressores e interferentes devem ser considerados.

\section{MATERIAL SUPLEMENTAR}

Espectros de absorção, de emissão e de fluorescência estão disponíveis gratuitamente em http://quimicanova.sbq.org.br, na forma de arquivo PDF.

\section{REFERÊNCIAS}

1. Mccormack, P.; Jones, P.; Hetherridge, M. J.; Rowland, S. J.; Water Res. 2001, 35, 3567

2. Somerville, H. J.; Bennett, D.; Daveport, J. N.; Holt, M. S.; Mar. Pollut. Bull. 1987, 18, 549.

3. Triggia, A. A.; Correia, C. A.; Clodoveu, V. F.; Xavier, J. A. D.; Machado, J. C. V.; Thomas, J. E.; Souza Filho, J. E.; Paula, J. L.; Rossi, N. C. M.; Pitombo, N. E. S.; Gouvêa, P. C. V. M.; Carvalho, R. S.; Barragan, R. V.; Fundamentos de Engenharia de Petróleo, 2a ed., Interciência: Rio de Janeiro, 2004.

4. Lima, R. M. G.; Wildhagen, G. R. S.; Cunha, J. W. S. D.; Afonso, J. C.; Quim. Nova 2008, 31, 1237.

5. http://pcc2526.pcc.usp.br/Arquivos/2007/Aulas/07.06.06\%20Aula14. pdf, acessada em Abril 2012.

6. http://www.rhodia.com/en/markets_and_products/product_finder/ product_details.tcm?keywords $=$ tolcide+ps \&productCode $=90016472 \&$ productName=TOLCIDE+PS+75, acessada em Abril 2012.

7. Oliveira, R. C. G.; Carvalho, C. H. M.; Oliveira, M. C. K.; Boletim Técnico Petrobras 2000, 43, 92.

8. Callaghan, D.; Baumgartner, W.; Proceedings SPE International Conference on Health, Safety, and Environment in Oil and Gas Exploration and Production, Houston, United States of America, 1990.

9. Brasil, Conselho Nacional do Meio Ambiente; Resolução $n^{\circ} 357$, de 17/3/2005, Diário Oficial da União, p. 1-23, 18/3/2005.

10. Knudsen, B.; Skjerve, S.; Frost, T. K.; Gangstad, A.; Proceedings $8^{\text {th }}$ SPE International Conference on Health, Safety, and Environment in Oil and Gas Exploration and Production, Abu Dhabi, United Arab Emirates, 2006.

11. Mcwilliams, P.; Payne, G.; Proceedings $7^{\text {th }}$ Oil Industry, Manchester, United Kingdom, 2001.

12. Castro, Y. E. V.; Dissertação de Mestrado, Pontifícia Universidade Católica do Rio de Janeiro, Brasil, 2006.

13. Sierra, M. M. S.; Giovanela, M.; Donard, O. F. X.; Belin, C.; Quim. Nova 1996, 19, 294. 
14. Skoog, D. A.; Holler, F. J.; Nieman, T. A.; Principles of Instrumental Analysis, $5^{\text {th }}$ ed., Harcout Brace College Publishing: New York, 1998.

15. Birks, J. B.; Photophysics of Aromatic Molecules, Wiley - Interscience: London, 1970.

16. Valeur, B.; Molecular Fluorescence: Principles and Applications, WileyVCH: New York, 2001.

17. Guilbault, G. G.; Practical Fluorescence, $2^{\text {nd }}$ ed., Marcel Dekker Inc.: New York, 1990, 265.

18. Larsson, T.; Wedborg, M.; Turner, D.; Anal. Chim. Acta 2007, 583, 357.

19. Esteves, V. I.; Santos, E. B. H.; Duarte, A. C.; J. Environ. Monit. 1999, $1,251$.

20. Settle, F. Em Handbook of Instrumental Techniques for Analytical Chemistry; Settle, F, ed.; Prentice-Hall: New Jersey, 1997, chap. 26.

21. Andre, J. C.; Bouchy, M.; Niclause, M.; Anal. Chim. Acta 1977, 92, 369.

22. Stevenson, C. L.; Vo-dinh, T.; Appl. Spectrosc. 1993, 47, 430.

23. Itoh, Y.; Horiuchi, S.; Yamamoto, K.; Photochem. Photobiol. Sci. 2005, 4, 835.

24. Fortenberry, C. L.; Grahmann, N. J.; Miller, C. D.; Son, A. J.; Proceedings SPE International Conference on Health, Safety, and Environment in Oil and Gas Exploration and Production, Houston, United States of America, 1993.
25. Minatti, E.; Tese de Doutorado, Universidade Federal de Santa Catarina, Brasil, 1999.

26. Migahed, M. A.; Mohamed, H. M.; Al-Sabagh, A. M.; Mater. Chem. Phys. 2003, 80, 169.

27. Son, A. J.; Muckleroy, B. S.; Proceedings the Nace Annual Conference and Corrosion Show, New Orleans, United States of America, 1997.

28. Maniasso, N.; Quim. Nova 2001, 24, 87.

29. Gonsalves, T. F. M.; Resumos do 16 Seminário de Iniciação Científica Puc-Rio, Rio de Janeiro, Brasil, 2008.

30. Roelants, E.; Schryver, F. C.; Langmuir 1987, 3, 209.

31. Sicilia, D.; Rubio, S.; Bendito, D. P.; Maniasso, N.; Zagatto, E. A. G.; Analyst 1999, 124, 615.

32. Son, A. J.; Chakravarty, J.; Proceedings the Nace Annual Conference and Corrosion Show, Denver, United States of America, 1996.

33. Lakowicz, J. R.; Principles of Fluorescence Spectroscopy, $3^{\text {rd }}$ ed., Springer: New York, 2006.

34. Kawakami, J.; Itoh, H.; Mitsuhashi, H.; Ito, S.; Anal. Sci. 1999, 15, 617.

35. Evangelista, J.; Tecnologia de Alimentos, Atheneu: São Paulo, 1994.

36. Jaffé, H. H.; Orchin, M.; Theory and Applications of Ultraviolet Spectroscopy, Wiley: New York, 1970. 


\section{ESTUDO DA EMISSÃO POR FLUORESCÊNCIA DE ULTRAVIOLETA NA QUANTIFICAÇÃO DE UM INIBIDOR DE CORROSÃO DO TIPO SAL DE AMÔNIO QUATERNÁRIO EM ÁGUA}

\section{Herllaine de Almeida Rangel*}

Instituto Estadual do Ambiente, Secretaria Estadual do Ambiente, Governo do Estado do Rio de Janeiro, Av. Salvador Allende, 5500, 22780-160 Rio de Janeiro - RJ, Brasil

\section{Fábio Merçon}

Instituto de Química, Universidade do Estado do Rio de Janeiro, Rua São Francisco Xavier, 524, Pavilhão Haroldo Lisboa da Cunha, 20550-013 Rio de Janeiro - RJ, Brasil

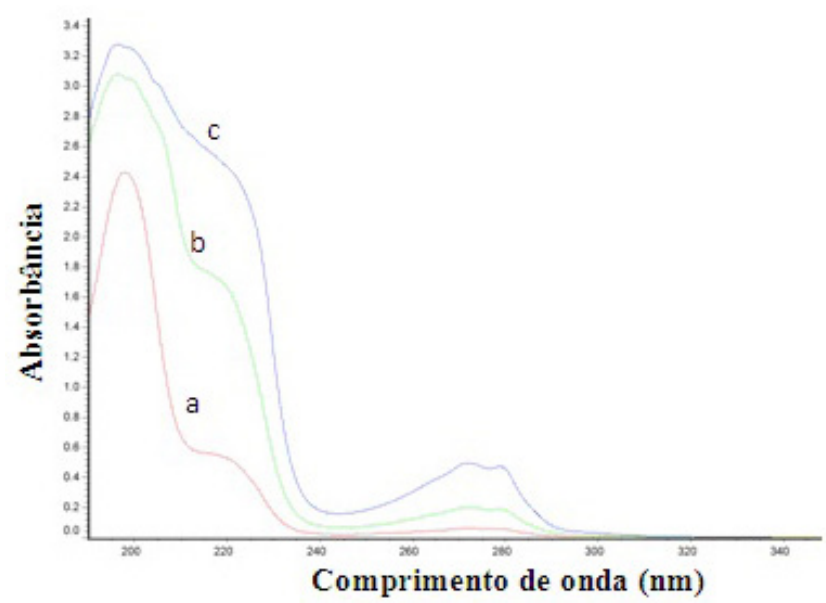

Figura 1S. Espectros de absorção de soluções aquosas de MDBTAC: (a) $30,0 \mathrm{mg} \mathrm{kg}^{-1}$; (b) 99,8 $\mathrm{mg} \mathrm{kg}^{-1}$; (c) 250,8 $\mathrm{mg} \mathrm{kg}^{-1}$

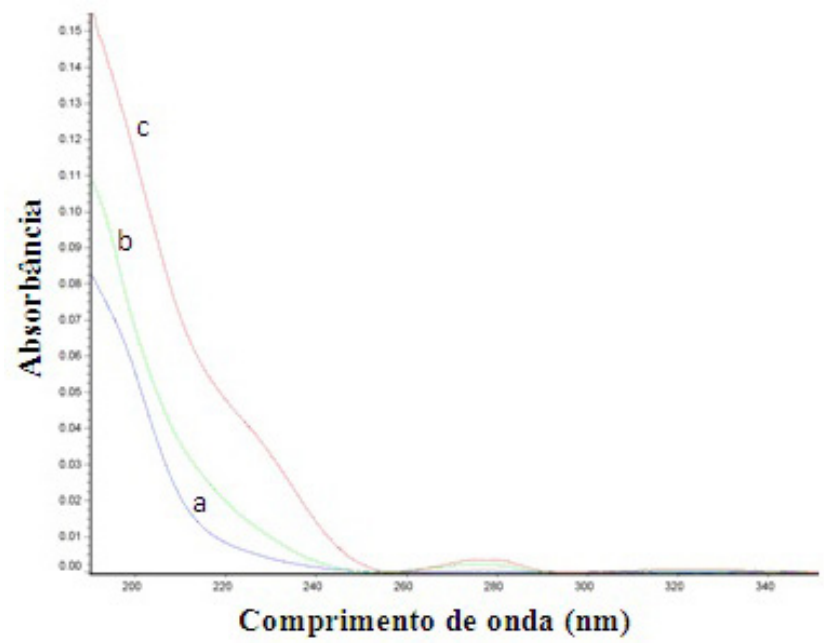

Figura 2 S. Espectros de absorção de soluções aquosas de DTAC: (a) $30,0 \mathrm{mg} \mathrm{kg}^{-1}$; (b) $100,1 \mathrm{mg} \mathrm{kg}^{-1}$; (c) $251,0 \mathrm{mg} \mathrm{kg}^{-1}$

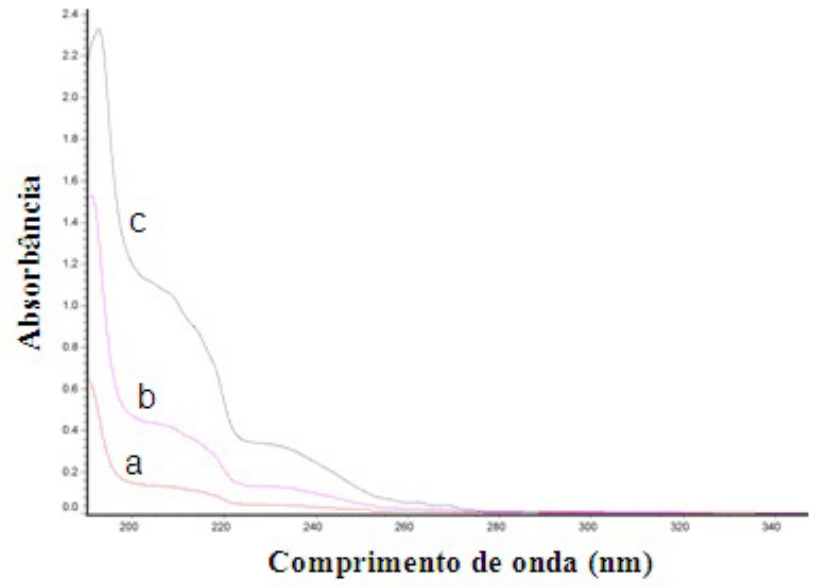

Figura 3S. Espectros de absorção de soluções aquosas de ICC: (a) 30,0 $\mathrm{mg} \mathrm{kg}^{-1}$; (b) 99,7 $\mathrm{mg} \mathrm{kg}^{-1}$; (c) 250,5 $\mathrm{mg} \mathrm{kg}^{-1}$

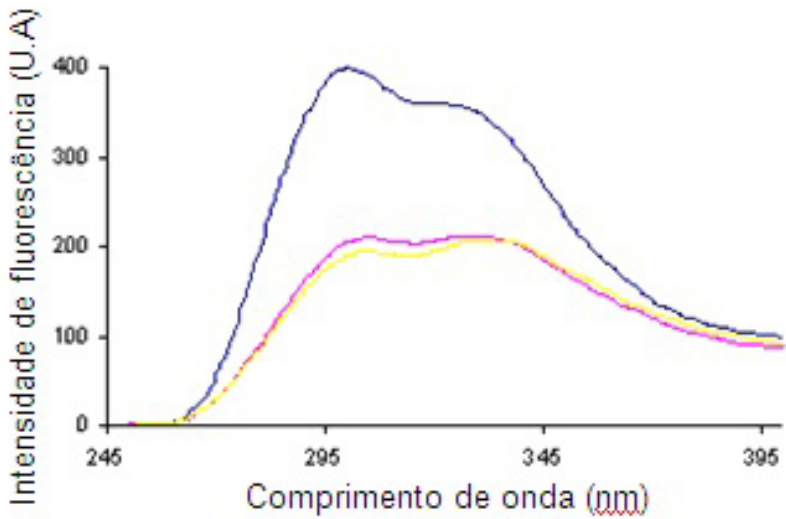

Figura 4S. Espectros de emissão $\left(\lambda_{e x}=230 \mathrm{~nm}\right)$ entre 245 e $395 \mathrm{~nm}$ de soluções de $10 \mathrm{mg} \mathrm{kg}^{-1}$ de inibidor de corrosão comercial em solução aquosa contendo 10\% metanol em função do tempo: -(dia 1), -(dia 2) e - (dia 3)

*e-mail: herllaine.almeida@inea.rj.gov.br 


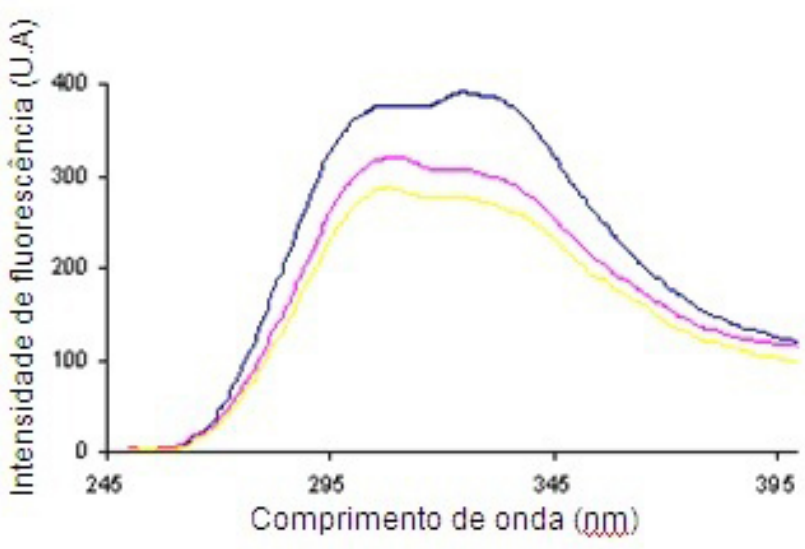

Figura 5S. Espectros de emissão $\left(\lambda_{e x}=230 \mathrm{~nm}\right)$ entre 245 e $395 \mathrm{~nm}$ de soluções de $10 \mathrm{mg} \mathrm{kg}^{-1}$ de inibidor de corrosão comercial em solução aquosa contendo 10\% isopropanol em função do tempo: -(dia 1), - (dia 2) e - (dia 3)

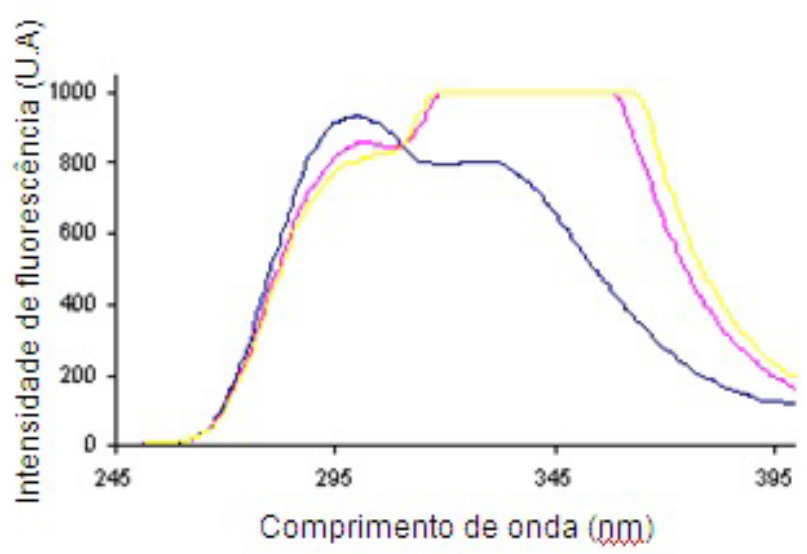

Figura 6S. Espectros de emissão $\left(\lambda_{e x}=230 \mathrm{~nm}\right)$ entre 245 e $395 \mathrm{~nm}$ de soluções de $10 \mathrm{mg} \mathrm{kg}^{-1}$ de inibidor de corrosão comercial em solução aquosa contendo $50 \%$ metanol em função do tempo: $-($ dia 1$),-($ dia 2$) e-($ dia 3$)$

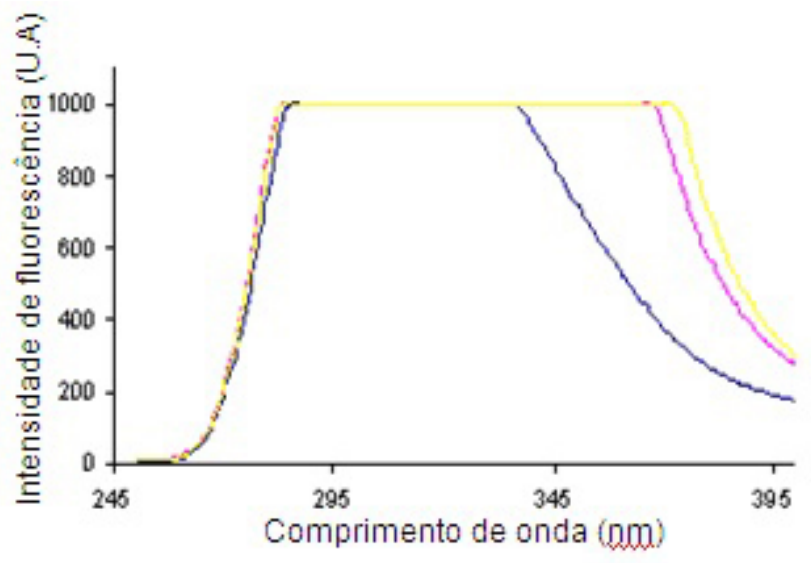

Figura 7S. Espectros de emissão $\left(\lambda_{e x}=230 \mathrm{~nm}\right)$ entre 245 e $395 \mathrm{~nm}$ de soluções de $10 \mathrm{mg} \mathrm{kg}^{-1}$ de inibidor de corrosão comercial em solução aquosa contendo $50 \%$ isopropanol em função do tempo: - (dia 1), - (dia 2) $e-($ dia 3$)$

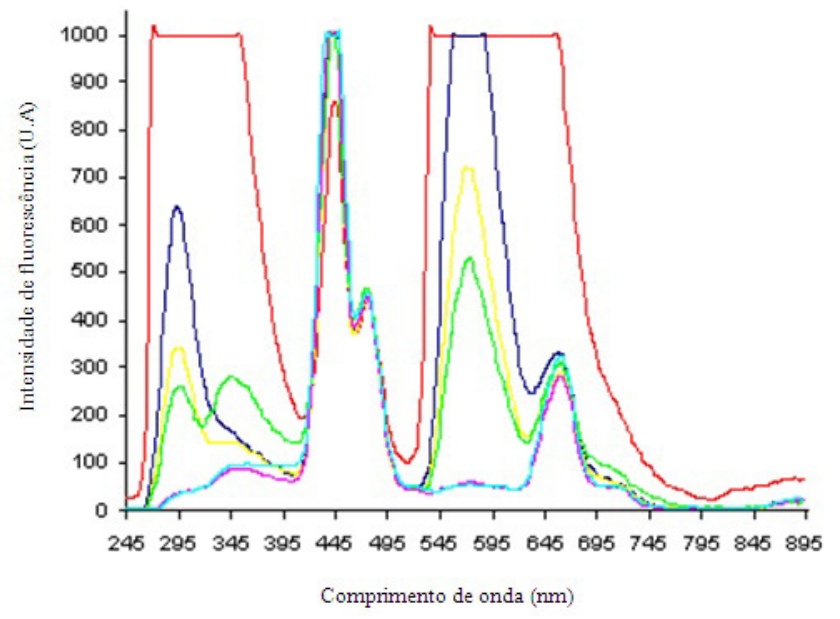

Figura 8S. Espectros de fluorescência de ultravioleta $\left(\lambda_{e x}=230 \mathrm{~nm}\right) \mathrm{em}$ água destilada (-) e de soluções com diferentes concentrações de biocida em água: $-1,01 \mathrm{mg} \mathrm{kg}^{-1}$; $-10,02 \mathrm{mg} \mathrm{kg}^{-1}$; $-100,3 \mathrm{mg} \mathrm{kg}^{-1}$; - 493,1 mg $\mathrm{kg}^{-1}$; . - 948,2 $\mathrm{mg} \mathrm{kg}^{-1}$; . 1939,5 $\mathrm{mg} \mathrm{kg}^{-1}$

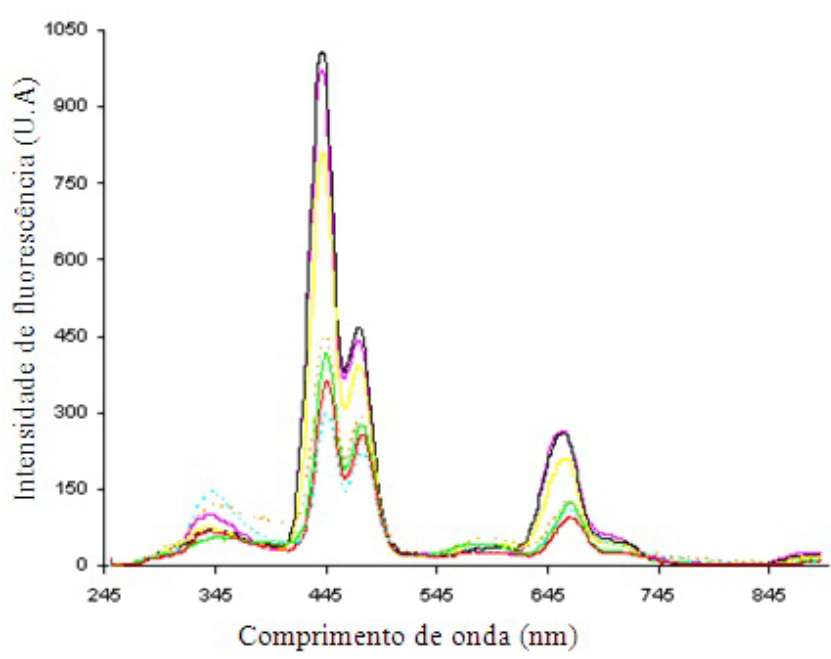

Figura 9S. Espectros de fluorescência de ultravioleta entre 245 e $395 \mathrm{~nm}$ de soluções com diferentes concentrações de biocida em água $\left(\lambda_{e x}=230 \mathrm{~nm}\right)$ : 9,8 $\mathrm{mg} \mathrm{kg}^{-1}$ de inibidor de corrosão (a); 9,8 $\mathrm{mg} \mathrm{kg}^{-1}$ inibidor de corrosão e 479,7 $\mathrm{mg} \mathrm{kg}^{-1}$ de biocida (b); 10,4 $\mathrm{mg} \mathrm{kg}^{-1}$ de inibidor de corrosão e 897,3 mg $\mathrm{kg}^{-1}$ de biocida (c) e 10,1 $\mathrm{mg} \mathrm{kg}^{-1}$ de inibidor de corrosão e $1951,7 \mathrm{mg}$ $\mathrm{kg}^{-1}$ de biocida $(\mathrm{d})$ 


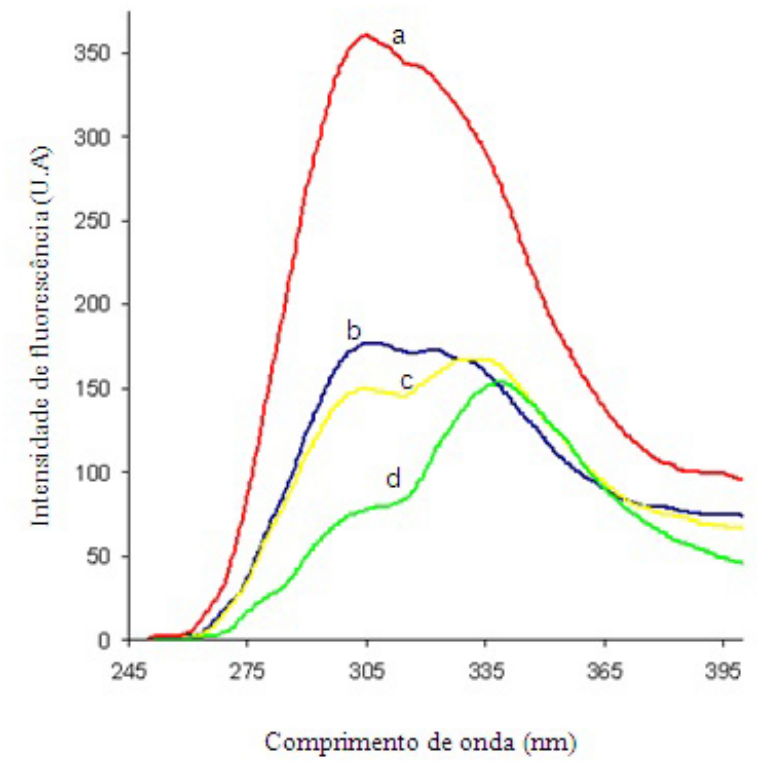

Figura 10S. Espectros de fluorescência de ultravioleta de soluções de BTEX em água $\left(\lambda_{e x}=230 \mathrm{~nm}\right): 526,3(-) ; 42,1(-) ; 21,1(-) ; 15,2(-) ; 1,235$ (一) $е$ 0,61 (-) $\mathrm{mg} \mathrm{kg}^{-1}$ 